\title{
Das Fiktionalitätsdilemma
}

\section{$\mathrm{Zu}$ einem poetologischen Problem und seiner historisch-narratologischen Auflösung}

\section{Hartmut Bleumer}

Zusammenfassung Ausgehend von der Beobachtung, dass sich die Literaturwissenschaft offenbar immer wieder ihres Fiktionalitätsbegriffs vergewissern muss, stellt die Skizze die Frage, ob diese Wiederholung nicht Ausdruck eines Dilemmas sein könnte, das die wissenschaftliche Begriffsrationalität im Umgang mit narrativen Strukturen selbst erzeugt. Als Lösungsvorschlag wird am Beispiel des Iwein Hartmanns von Aue darauf hingewiesen, dass der erste programmatisch fiktionale Artusroman dieses Dilemma im Erzählen am Beispiel des Weltverständnisses seines Protagonisten vorführt und ästhetisch auflöst: in der paradoxen Korrelation narrativer Ebenen, die es einer historischen Narratologie erlauben müsste, die metaphorische Ästhetik des Artusromans genauer zu erschließen.

Schlüsselwörter Fiktionalität · Historische Ästhetik · Historische Narratologie · Metapher · Hartmann von Aue $\cdot$ Iwein 


\title{
The Dilemma of Fictionality
}

On a Poetological Problem and its Resolution in Historical Narratology

\begin{abstract}
Based on the observation that literary studies must apparently constantly reassert its concept of fictionality, the sketch poses the question of whether this repetition might not be the expression of a dilemma that scientific conceptual rationality itself creates when dealing with narrative structures. As a proposed solution, it is pointed out, using the example of Hartmann von Aue's Iwein, that the first programmatically fictional Arthurian novel presents and aesthetically resolves this dilemma in narrative, using the example of its protagonist's understanding of the world: in the paradoxical correlation of narrative levels, which should allow a historical narratology to more precisely develop the metaphorical aesthetics of the Arthurian novel.
\end{abstract}

Keywords Fictionality · Historical Aesthetics · Historical Narratology ·

Metaphor $\cdot$ Hartmann von Aue $\cdot$ Iwein

\section{Ein Begriff und sein iterativer Effekt im Erzählen}

Die Frage nach dem Anfang, den Bedingungen und den Folgen der Fiktionalität ist ein ständig wiederkehrendes Phänomen der Literaturwissenschaft. ${ }^{1}$ Dabei scheint es fast so, als müsse die Fiktionalität immer wieder neu entdeckt und diskutiert werden. Dies gilt gerade auch in historischer Hinsicht. So hat man im Rahmen der Vorstellung einer großen literaturgeschichtlichen Entwicklung seit den 1980erJahren von der »Entdeckung der Fiktionalität « durch den arthurischen Romans um 1200 gesprochen. ${ }^{2}$ Was damit aber genau gemeint sein könnte, blieb durchaus offen. Immerhin hätte eine solche Entdeckung insofern historisch trivial und damit bildungsgeschichtlich alltäglich sein müssen, als die Fabel als das einfachste, klassischrhetorische Beispiel der fictio und ihrer Selbstreferentialität schon im Schulunterricht des Mittelalters gelten darf. Entsprechend konnte die Kritik aus Sicht der an die Rhetorik anschließenden, lateinischen Gattungspoetik nicht ausbleiben. ${ }^{3}$ Angezielt war mit dieser These also offenbar etwas Anderes, an dem die ästhetisch inter-

\footnotetext{
1 Vgl. den Querschnitt bei Klauk, Tobias/Köppe, Tilmann (Hg.): Fiktionalität. Ein interdisziplinäres Handbuch. Berlin/Boston 2014. Zum historischen Problemhorizont bes. Franzen, Johannes u.a.: »Geschichte der Fiktionalität. Zur Einleitung«. In: Ders. u. a. (Hg.): Geschichte der Fiktionalität. Diachrone Perspektiven auf ein kulturelles Konzept. Baden-Baden 2018, S. 7-18, hier 7-14.

2 Haug, Walter: »Die Entdeckung der Fiktionalität«. In: Ders.: Die Wahrheit der Fiktion. Studien zur weltlichen und geistlichen Literatur des Mittelalters und der frühen Neuzeit. Tübingen 2003, S. 128-144.

3 Vgl. die an der lateinischen Rhetorik und Poetik ausgerichtete Kritiken der mediävistischen Fiktionalitätsdebatte in der Reihe der Arbeiten von Fritz Peter Knapp, bes. Knapp, Fritz Peter: Historie und Fiktion in der mittelalterlichen Gattungspoetik. Sieben Studien und ein Nachwort. Beiträge zur älteren Literaturgeschichte. Heidelberg 1997. Ders.: Historie und Fiktion in der mittelalterlichen Gattungspoetik: 2. Zehn neue Studien und ein Vorwort. Heidelberg 2005. Weiterführend zum Schwanken zwischen andersweltlichmythischem und märchenhaft-antirealistischem Erzählen im Artusroman den Lösungsvorschlag über die idealisierende Axiologie des Märchens bei Knapp, Fritz Peter: Die Geburt des fiktionalen Romans aus dem Geiste des Märchens. Vorgetragen am 25. Oktober 2013. Heidelberg 2014, S. 25-32.
} 
essierte Forschung weiter festgehalten hat. Und für dieses Andere ist zunehmend deutlich geworden, dass es unter den Bedingungen der älteren Medialität und ihrer Präsenzsemantik im differenzlogischen Fiktionalitätsbegriff noch nicht aufgeht. ${ }^{4}$

Dessen ungeachtet hat man die Geltung des Fiktionalitätsbegriffs mit eigentümlicher Entschiedenheit auch noch dort behauptet, wo er für die neuere Literatur als strittig gilt: mit Blick auf die mittelhochdeutsche Lyrik, genauer den Minnesang des 12. und 13. Jahrhunderts. ${ }^{5}$ Die seit Beginn der 2000er Jahre geführte Diskussion um die Fiktionalität des Minnesangs hat gleichwohl auch hier nicht nur zu wichtigen Klärungen der performativen Medialität der Lyrik geführt, sie hat ebenso erneut jene Geltungsbehauptung herausgefordert, die mit der Fiktionalitätshypothese offenbar untrennbar verbunden ist. Vielleicht wird diese unter den ästhetischen Bedingungen der Lyrik sogar noch stärker spürbar als im Rahmen der Narrativik, nur vermag der Minnesang seine textuelle Fiktion und seine fiktionale Textualität im performativen Akt des Sanges auch aufzugeben, ${ }^{6}$ womit letztlich auch das terminologische Grundproblem in den Hintergrund tritt. »Minnesang ist fiktional «: ${ }^{7}$ Das ist texttheoretisch ebenso richtig wie performanztheoretisch falsch - nur ist der Kurzschluss der apodiktischen Fiktionalitätsbehauptung nicht mehr wichtig, wenn das Spiel mit den Fiktionen ästhetisch zur Realität wird.

Eine Schwierigkeit ist freilich nicht schon dadurch erledigt, dass sie in den Hintergrund tritt, vielmehr flackert die Debatte um das Stichwort gerade dadurch immer wieder auf. ${ }^{8}$ Denn in der Lyrik wie in der Narrativik scheint die offenkundige, wissenschaftliche Attraktivität des Fiktionalitätsbegriffs, zunächst für die ältere Literatur gesagt, nur bedingt geeignet zu sein, jene ästhetische Attraktivität zu erklären, die der Terminus wissenschaftlich bannen soll. Das in diesem Effekt angedeutete Dilemma

\footnotetext{
4 Als jüngste Bilanz Häger, Hanna-Miriam: Fiktionalität trans- und intermedial. Arthurische Möglichkeitsräume in Mittelalter und Moderne. Wiesbaden 2019, S. 23-46. Vgl. zuvor besonders die Resümees und Voten von Glauch, Sonja: »Fiktionalität im Mittelalter; Revisited «. In: Poetica 46 (2014), S. 85-139. Dies.: »Fiktionalität im Mittelalter«. In: Klauk/Köppe (s. Anm. 1), S. 385-418.

5 Vgl. apodiktisch »Minnesang ist fiktional«, Müller, Jan-Dirk: »Die Fiktion höfischer Liebe und die Fiktionalität des Minnesangs«. In: Albrecht Hausmann (Hg.): Text und Handeln. Zum kommunikativen Ort von Minnesang und antiker Lyrik. Heidelberg 2004, S. 49-64, hier S. 49, dessen kritische Modifikationen die rhetorisch in Frage gestellte Fiktionalitätsprämisse gerade festgeschrieben haben; ders.: »Literarische und andere Spiele. Zum Fiktionalitätsproblem in vormoderner Literatur«. In: Poetica 36 (2004), S. 281-311. Dass die Infragestellung dieser Prämissen durch Haferland, Harald: Hohe Minne. Zur Beschreibung der Minnekanzone. Berlin 2000, S. 27-32, zu einer produktiven Verunsicherung geführt haben, sei festgehalten, zumal ihr zentrales Argument in der weiteren Diskussion nicht völlig klar geworden ist.

6 Vgl. Warning, Rainer: »Der inszenierte Diskurs. Bemerkungen zur pragmatischen Relation der Fiktion«. In: Dieter Henrich/Wolfgang Iser (Hg.): Funktionen des Fiktiven. München 1983, S. 183-206, hier S. 193. Strohschneider, Peter: »>Nu sehent, wie der singet!< Vom Hervortreten des Sängers im Minnesang«. In: Jan-Dirk Müller (Hg.): »Aufführung« und »Schrift« in Mittelalter und Früher Neuzeit. Stuttgart/Weimar 1996, S. 7-30. Bleumer, Hartmut: »Ritual, Fiktion und ästhetische Erfahrung. Wandlungen des höfischen Diskurses zwischen Roman und Minnesang«. In: Ruth Florack/Rüdiger Singer (Hg.): Die Kunst der Galanterie. Facetten eines Verhaltensmodells in der Literatur der Frühen Neuzeit. Berlin/New York 2012, S. 51-92.

7 Müller (s. Anm. 5).

8 Vgl. die Beiträge in Peters, Ursula/Warning, Rainer (Hg.): Fiktion und Fiktionalität in den Literaturen des Mittelalters. Jan-Dirk Müller zum 65. Geburtstag. Paderborn 2009. Speziell zum Artusroman der Band von Przybilski, Martin/Ruge, Nikolaus (Hg.): Fiktionalität im Artusroman des 13. bis 15. Jahrhunderts. Romanistische und germanistische Perspektiven. Wiesbaden 2013.
} 
setzt sich sogar historisch-systematisch fort: Ganz ähnlich wie in der germanistischen Mediävistik ruft die Fiktionalität nämlich auch nach der modernen Wende zum technischen Möglichkeitsdenken gleichsam nach ihrer Beachtung als endgültig zu fixierendem, zentralen wissenschaftlichen Leitbegriff. ${ }^{9}$ Nur entpuppt sie sich dabei erneut als ein Problem, das ebenso für jede Wissenschaftsphase gesichert werden muss, wie es auch jede Literaturwissenschaftlerin und jeder Literaturwissenschaftler für sich zu klären hat. Fiktionalität wäre damit ein historischer Begriff, der sich seinem eigenen terminologischen Anspruch zum Trotz einer (historischen) Fixierung widersetzt.

Die Vermutung der folgenden Skizze lautet, dass es diese begriffliche Schwierigkeit sein könnte, die im modernen Konzept der Fiktionalität selbst, nämlich in ihrer epistemischen, irreduziblen Angewiesenheit auf die Faktizität begründet liegt. Der seit den rhetorischen Anfängen latent wirksame Gegenbegriff des Faktums erzeugt dabei insbesondere im Erzählen fortgesetzt jenes Dilemma, das die wissenschaftliche Betrachtung aufzulösen versucht. Nur wird es durch die wissenschaftliche Betrachtung auch immer wieder hergestellt. Denn das Erzählen verhandelt die Fiktion gerade nicht als Dilemma, sondern als Paradoxie.

Historisch wird diese Verhandlung schon im Erzählen selbst vorgeführt, nämlich ausgerechnet schon in jenem Text, der als literaturhistorischer Kronzeuge für die sogenannte Entdeckung der Fiktionalität ins Feld geführt worden ist: dem Iwein Hartmanns von Aue als erstem programmatischen Aventiureroman. ${ }^{10}$ Dieser Roman macht sogar klar, worin jenes Dilemma besteht, das in der modernen Fiktionalitätsthese nicht nur wiederentdeckt wird, sondern dem gegenüber das Erzählen immer schon seine ästhetische Überlegenheit ausspielt.

\section{Im Zirkel des Erzählens}

Hartmanns Roman ist gleich in mehrfacher Hinsicht ein >Wiedererzählen $<.{ }^{11}$ Es handelt sich sowohl um eine Interpretation der Vorlage von Chretiens Yvain ou Le Chevalier au Lion als auch um eine »Interpretation der Erzählung durch die

\footnotetext{
9 Vgl. Kablitz, Andreas: Kunst des Möglichen. Theorie der Literatur. Freiburg/Berlin/Wien 2013, bes. S. 197-204.

10 Vgl. zur Paradoxie der Aventiure am deutlichsten das ihr methodisch angemessene close reading von Geisthardt, Constanze: Monster als Medien literarischer Selbstreflexion. Untersuchungen zu Hartmanns von Aue Iwein, Heinrichs von dem Türlin Crône und Johanns von Würzburg Wilhelm von Österreich. Berlin 2019, S. 95-104, mit souveräner Forschungsübersicht sowie den Forschungsnachweisen, und für das Folgende Bleumer, Hartmut: »Fee, Ereignis und Sujet. Zu einem narratologischen Begriffsproblem am Beispiel des >klassischen< Artusromans«. In: Michael Schwarzbach-Dobson/Franziska Wenzel (Hg.): Aventiure. Ereignis und Erzählung. Berlin (im Druck).

11 Worstbrock, Franz Josef: »Wiedererzählen und Übersetzen«. In: Walter Haug (Hg.): Mittelalter und frühe Neuzeit. Übergänge, Umbrüche und Neuansätze. Tübingen 1999, S. 128-142. Als Grundlage des Ansatzes Worstbrock, Franz Josef: »Dilatatio materiae. Zur Poetik des >Erec $<$ Hartmanns von Aue «. In: FmSt 19 (1985), S. 1-30. Die verallgemeinernde Erweiterung durch das Stichwort >Retextualisierung< unterstreicht die Relevanz des Begriffs, wenn sie auch die mögliche historisch-narratologische Pointe notwendig erheblich abschwächt. Vgl. das Spektrum der Beiträge in Bumke, Joachim/Ursula Peters (Hg.): Retextualisierung in der mittelalterlichen Literatur. Berlin 2005.
} 
Erzählung «, ${ }^{12}$ in der dann noch einmal -wie der französische Titel bereits anzeigt der Protagonist durch das Bild des Ritters mit dem Löwen interpretiert wird. Der damit schon angedeutete, mehrfach durchlaufene Prozess der narrativen Selbstinterpretation beinhaltet somit einen Zirkel, den man gerade in der Fiktionalitätsdiskussion als ein Moment des Spiels charakterisiert hat. ${ }^{13}$ Diese spielerische Anmutung ist aber nicht zuletzt dem Umstand geschuldet, dass die entsprechende wissenschaftliche Diskussion zugleich die Spiele des Erzählens zu fixieren versucht, und zwar in einem Begriff von Wirklichkeit oder Faktizität, den sie zwar durchstreicht, der aber gerade dadurch permanent in ihrer Praxis anwesend ist.

Dass schon der Iwein-Prolog deutlich macht, Artus sei längst gestorben, aber in der Erinnerung und im Erzählen lebe sein Name weiter (Iw 8-17), ${ }^{14}$ bezeugt indes durchaus keine antithetische Denkfigur in dem Sinne: die Fakten sind tot, es lebe die Literatur. Oder weniger polemisch ausgedrückt: Es geht nicht um den »Gegensatz von Literatur und Leben«, über den jene »Überlegenheit der Literatur über die bloße Faktizität [...] damit zum erstenmal explizit formuliert « sei, die man auf den Namen der Fiktionalität getauft hat. ${ }^{15}$ Auch ist die Fiktionalität an sich längst nicht mehr zu entdecken. Was im Iwein Hartmanns vielleicht für das Mittelhochdeutsche erstmalig ausgesprochen wird, kennt im Lateinischen buchstäblich längst jedes Kind, das es mit Fabeln zu tun hatte. Die Entdeckung, um die es geht, gilt so recht eigentlich nicht der Fiktionalität. Und doch kommt es tatsächlich im fiktionalen Roman zu einer aufsehenerregenden Entdeckung. Sie wird durch die Fiktionalität ermöglicht. Sie gilt dem Kernmechanismus des Erzählens, der die Fiktionen ständig in ihrer spielerischen Bewegung und damit am Leben hält.

Der methodische Unterschied dieser narrativen Spielfigur, in der die Narration gleichsam mit dem Erzählen wie mit dem Erzählten jongliert, ${ }^{16}$ zum wissenschaftlich zunächst postulierten Fiktionalitätskonzept liegt darin, dass sie in dynamischen Korrelationsbeziehungen denkt, wo die Fiktionalitätsfrage automatisch in Oppositionen und Hierarchieebenen feststeckt. Das Ziel der Fiktionalitätsfrage ist es nämlich, dem Spiel einen faktischen Grund zu geben, von dem es sich abstoßen kann. Das Ziel des Erzählens scheint dagegen in seinem Spiel selbst auf. In diesem Spiel ist die Fiktionalität nicht der Zweck der literarischen Entdeckungen, in diesem Spiel ist die Fiktionalität ihr Mittel.

\footnotetext{
12 Kern, Peter: »Interpretation der Erzählung durch Erzählung. Zur Bedeutung von Wiederholung, Variation und Umkehrung in Hartmanns >Iwein««. In: ZfdPh 92 (1973), S. 338-359.

13 Vgl. Haug, Walter: »Chrétiens >Yvain< und Hartmanns >Iwein<: Das Spiel mit dem arthurischen Modell«. In: Ders.: Die Wahrheit der Fiktion. Studien zur weltlichen und geistlichen Literatur des Mittelalters und der frühen Neuzeit. Tübingen 2003, S. 223-238. Zuerst unter dem die ästhetischen Effekte der reflexiven Brüche noch stärker akzentuierenden Titel: »Das Spiel mit der arthurischen Struktur in der Komödie von Yvain / Iwein«. In: Friedrich Wolfzettel (Hg.): Erzählstrukturen der Artusliteratur. Forschungsgeschichte und neuerer Ansätze. Unter Mitwirkung von Peter Ihring. Tübingen 1999, S. 99-118.

14 Ausgabe: Hartmann von Aue: »Iwein«. In: Ders.: Gregorius. Der arme Heinrich. Iwein. Hrsg. und übersetzt von Volker Mertens. Frankfurt a. M. 2008, S. 317-767.

15 Haug, Walter: Literaturtheorie im deutschen Mittelalter. Von den Anfängen bis zum Ende des 13. Jahrhunderts. 2., überarbeitete und erweiterte Auflage. Darmstadt 1992, S. 126.

16 Vgl. Mertens, Volker: »Theoretische und narrativierte Narratologie von Chrétien bis Kafka«. In: Harald Haferland/Matthias Meyer (Hg.): Historische Narratologie. Mediävistische Perspektiven. Berlin/New York 2010, S. 17-34.
} 
Umständlich theoretisch formulieren muss man diesen grundsätzlichen poetischen Primat gegenüber den vielen, praktisch so erfolgreichen Interpretationen des Iwein eigentlich kaum. Darum ist es interessant, dass er gerade in der lange florierenden, theoretisch orientierten Fiktionalitätsdebatte Schwierigkeiten gemacht hat. Forschungsgeschichtlich hängt das Problem, wie oft betont wurde, speziell für die arthurische Poetik mit der Fixierung auf die sogenannte >gestufte Doppelwegstruktur< zusammen, die nicht nur narratologisch einem durchaus eigenwilligen Strukturbegriff folgt, sondern die auch unter dem Einfluss der Fiktionalitätsdebatte die methodische Potenz der oben bereits zitieren, unangestrengten Grundformel von Peter Kern zur >Interpretation der Erzählung durch die Erzählung<verdeckt hat. Das Folgeproblem ist ebenso lange bekannt: Kennt man den gestuften Doppelweg als prägendes Kompositionsmodell, dann wird der Erzähltext zum toten Klassiker. Die Interpretation hat ihren festen Weg und verliert eben jenen spielerischen Charakter, den das Modell im Weg des Verstehens eigentlich garantieren soll. ${ }^{17}$

Diese Stillstellung der narrativen Dynamik ist aber nicht allein durch die sog. >Doppelwegstruktur < bedingt. Sie beruht vielmehr forschungsgeschichtlich auf einer stillschweigenden Allianz ihres Ordnungsprinzips mit dem Fiktionalitätsbegriff, der ein Spiel verspricht, das er selbst kategorisch stillstellt. Fast möchte man sagen: Fiktionalitätstheoretiker sind Spielverderber. Setzt man dagegen noch einmal an der - wenn man so will - narrativen >Kern-Formel< an, dann ergibt sich ein nichthierarchisches Verständnis, durch das man hoffen kann, das Fiktionalitätsdilemma aufzulösen.

So eröffnet der Erzähler des Iwein seine Narration damit, dass am Artushof eine Figur von dem erzählt, was ihr einst widerfahren sein soll. Dieses erzählte Widerfährnis wirkt noch weit fabulöser, als es der Artushof in seiner zweifelhaften Überidealisierung ohnehin schon ist, und doch schickt sich der Protagonist im blinden Glauben an den Realitätsgehalt dieser Erzählung an, sie praktisch selbst in eine Geschichte umzusetzen, d.h. die narrative Fiktion Realität werden zu lassen. In dieser so verstandenen Geschichte geht es Iwein darum, sich einen Namen zu machen. Eine Figur versucht also, ihrem eigenen narrativen Entwurf folgend, sich durch ihre Geschichte eine eigene Erzählung zu geben. Das grandiose Scheitern dieses narrativen Selbstentwurfes führt aber nicht etwa aus dem Erzählen heraus, es führt erst richtig in das Erzählen hinein. Es mündet in einen paradoxen narrativen Zirkel, der sich poetisch als alternativlos erweist.

Als Modellphase für diesen geradezu schwindelerregenden Zirkel darf die Phase von Iweins Wahnsinn gelten, ihre Zentralstelle ist das viel diskutierte Ereignis von Iweins Erwachen. Der metapoetische Aufschlusswert gerade dieser Szene dürfte nicht zu unterschätzen sein, weil die Episode letztlich zeigt, dass der Roman insgesamt einen durchgängig metaphorischen Charakter hat. Die genaueren Implikationen der Stelle können zur Erörterung des Erzählproblems, das diese Skizze lediglich kurz andeuten möchte, beiseite bleiben. Zu erwähnen ist nur: Iweins Traum, aus dem er zu erwachen meint, ist letztlich die metapoetische Metapher für den imaginären Status der Literatur und ihrer Fiktionen.

17 Vgl. in diesem Sinne die viel zitierte Polemik von Schmid, Elisabeth: »Weg mit dem Doppelweg. Wider eine Selbstverständlichkeit der germanistischen Artusforschung«. In: Wolfzettel (s. Anm. 13), S. 69-85. 
Der Traum, aus dem Iwein durch sein Erwachen in die Wirklichkeit zurückkehrt, eröffnet in der Literatur die Augen dafür, dass schon diese einen grundsätzlich imaginären Status hat. Fiktionalitätstheoretisch heißt das: Die Imagination ist nicht, wie in den modernen Fiktionalitätstheorien angedeutet, der Fiktion nachgeordnet, ${ }^{18}$ sie ist ihre ästhetische Voraussetzung. Sie ist der eigentliche Ort der Immersion in die poetischen Welten. ${ }^{19}$ Darum ist auch die Fiktionalität nur ein Mittel, jenen Sinn zu erschließen, den narrative Prozesse in der Imagination erzeugen.

Entsprechend wird Iwein nach seinem Wahnsinn zu einem Ritter mit dem Löwen, zu einem namenlosen, rätselhaften Bildereignis. Iwein ist, wo er sich in Szene setzt, ebenso eine handelnde Figur wie eine dezente Metapher für das Erzählproblem; in ihm wird der Glaube an ein ritterliches Ideal poetisch Wirklichkeit, weil das Modell dieser Wirklichkeit in den Zirkel des Erzählens hineingeholt wurde. Mehr noch: Im Erzählen entdeckt die Wahrnehmung von Wirklichkeit ihren eigenen imaginären Zirkel.

Indiz dieses Zirkels ist nicht zuletzt die Begegnung Iweins mit dem Löwen, aus der die Verwandlung Iweins zum Löwenritter hervorgeht (Iw 3825-3882). Denn in dem Maße, wie die Figur Iweins über den Löwenritter zum Bild, d.h. der figura des idealen Ritters wird, wandelt sich umgekehrt der Löwe vom wandelnden Wappentier zur menschlichen persona. ${ }^{20}$ So ist die Situation, in der Iwein auf die beiden gegeneinander kämpfenden Tiere, den Drachen und den Löwen trifft, eine erkennbar metaphorische. Iwein steht mit der Frage, welchem Tier er beistehen soll, vor einer bildlichen Scheidewegsituation, die dem Protagonisten mit der Entscheidung, [...] daz er wolde / helfen dem edelen tiere (3848f.), die ritterliche Variante eines heroischen Drachenkampfes beschert.

Dabei wird die Entscheidung für den Löwen handlungslogisch als ein Dilemma formuliert. Denn der Löwe ist nicht weniger gefährlich als der Drache, den Drachen zu töten müsste also praktisch bedeuten, dass sich der Löwe gegen seinen Retter wendet (Iw 3850-3864). Doch das Dilemma löst sich auf, weil der Löwe zwar - wie gesagt - edel, aber nicht - wie erwartet - ein wildes Tier ist. Der Löwe erweist sich als ein Musterbild der feudalen Axiologie, er verhält sich dem adligen Dienstideal entsprechend und erkennt Iwein nach seiner Rettung als Herrn an. Und als wäre der nun folgende treue Dienst des Löwen an dem Ritter nicht schon merkwürdig genug, führt diese Treue schon kurz darauf bis in einen äußerst menschlich gedachten Tod: Als Iwein in Erinnerung an seine Verfehlung, die ihn schon einmal in Wahn und

18 Iser, Wolfgang: Das Fiktive und das Imaginäre. Perspektiven literarischer Anthropologie. Frankfurt a. M. 1991. Herweg, Mathias: Wege zur Verbindlichkeit. Studien zum deutschen Roman um 1300. Wiesbaden 2010, S. 19-21, 433f.

19 Vgl. so zum Iwein bereits Bleumer, Hartmut: »Von der Fiktion zur Immersion. Narrative Semantik und ästhetische Erfahrung im >Wigalois< des Wirnt von Grafenberg «. In: Martin Przybilski/Nikolaus Ruge (Hg.): Fiktionalität im Artusroman des 13. bis 15. Jahrhunderts. Romanistische und germanistische Perspektiven. Wiesbaden 2013, S. 83-105, hier S. 86f.

${ }^{20}$ Der mediävistische Forschungsstand zum Verhältnis der Begriffe von Figur, Person und Persona ist schwer zu ermitteln. Als Ausgangspunkte künftig unerlässlich sind die umsichtig recherchierten Arbeiten von Möllenbrink, Linus: Person und Artefakt. Zur Figurenkonzeption im >Tristan< Gottfrieds von Straßburg. Tübingen 2020, und Zudrell, Lena: Historische Narratologie der Figur. Studien zu den drei Artusromanen des Pleier. Berlin/Boston 2020, zwischen deren methodisch unterschiedlichen Zugriffen sich ein eigenes Forschungsfeld abzeichnet. 
Ohnmacht geführt hatte, an der Gewitterquelle, mit der jener metaphorische Aventiureweg begann, der in den Traumbildern des Wahns auf seinen metapoetischen, metaphorischen Begriff kommt, erneut ohnmächtig wird und wie tot vom Ross fällt, geschieht höchst Seltsames.

Schon Iwein fällt hier nicht einfach vom Pferd. Vielmehr löst sich seine ritterliche Waffe, das Symbol seines Rittertums, bei dem Sturz aus der Scheide, und der Ritter fällt in sein eigenes Schwert. Angesichts des nunmehr in seinem Blut wie tot am Boden liegenden Iwein gerät der Löwe in solche Not, dass er sich, ebenfalls durch das Schwert Iweins, selbst zu töten versucht. Erst im letzten Moment kehrt das Leben in Iwein zurück, so dass dieser den Löwen von seinem Selbstmord abhalten kann.

Die Szene mit dem suizidalen Löwen, der das Schwert seines Herren eigens in einem Strauch ausrichtet, um sich hineinzustürzen, ist sehr kurz, sie ist in dieser Kürze vielleicht sogar etwas witzig, in jedem Falle aber durchaus komisch, und doch ist sie mehr als nur ein Fiktionssignal. Sie setzt nämlich nochmals eben jene Metapher ins Bild, mit welcher der Erzähler den Wahnsinn Iweins schon hatte beginnen lassen. Denn die berühmte Stelle lautet dort:

Er verlôs sîn selbes hulde:

wan ern mohte die schulde

ûf nieman anders gesagen:

in hete sîn selbes swert erlagen.

er nahte weder man noch wîp,

niuwen $\hat{u} f$ sin selbes lîp.

Er stal sich swîgende dan

[...] (Iw 3220-3227)

So wie Iwein sich metaphorisch gesprochen durch sein eigenes Schwert zu Fall gebracht hat, indem er um der falsch verstandenen Âventiure willen vergaß, zu seiner Königin zurückzukehren, so erinnert er sich hier des Vergessens und fällt tatsächlich in sein Schwert. Die Metapher des Diskurses realisiert sich in der Handlung. Dass nun auch sein Löwe, die in der histoire bzw. der Diegese real gewordene Metapher des edlen Rittertums, es dem Ritter hier tatsächlich gleichtun will, macht vollends deutlich, dass die Realität dieser Erzählwelt immer auch eine Metapher ist. Und in diesem metaphorischen Verständnis kann der Löwe zur persona, zur sichtbaren Maske der Iweinfigur werden.

\section{Schlussfolgerungen: Die zwei Seiten der Erzählung}

Was hier emblematisch für das Erzählte sichtbar wird, gilt damit auch für das Erzählen generell. Denn allgemeiner heißt das: Was immer den Figuren im Iwein als vermeintlich wirkliches Geschehen begegnet, ist in der Figur als figura oder Bild immer schon eine Metapher. Diese Tropologie des Erzählten gilt es nur zu entdecken und dramatisch auszuspielen. Für den Übergang der modernen Narratologie zu einer historischen, die sich an der Strukturdiskussion des Artusromans und seines 
Fiktionalitätsproblems zu orientieren hat, bedeutet diese tiefgreifende, konkrete Metaphorisierung eine recht grundsätzliche Herausforderung. Denn es gibt in diesem Erzählen keine einfache Ebenenhierarchie, wie sie zuerst im rhetorischen Verständnis der wirklichkeitsorientierten Gerichtsrede mit ihrer realistischen Epistemologie angelegt ist und dann im modernen narratologischen Modell der Erzählebenen wiederkehrt. Es gibt also, um bei der Rhetorik zu bleiben, keine Sinnkonstitution, die mit den Fakten beginnt, diese gedanklich-kompositorisch ordnet und dann nur noch sprachlich gestaltet und metaphorisch ausschmückt. Die Gedankenfiguren und Tropen, die hier am Ende der Redekonzeption stehen, begegnen in der Poesie schon an ihrem Anfang. Die Tropen bilden keine Alternative zum Geschehen der vermeintlichen Wirklichkeit, sie sind ihr von Anfang an eingeschrieben.

Für das historisch-narratologische Verständnis, das nicht umhin kann, sich über ein Modell von Erzählebenen zu orientieren, führt eben diese grundlegende Metaphorizität zu ihrer typischen Korrelationsbeziehung. ${ }^{21}$ In ihr verlaufen die Ebenen nicht einfach im Kreis, weil das Spiel des Erzählens auf der höchsten Ebene der Narration als Erzählgeschehen von vorn beginnen kann. Die Hierarchien dieses Modells, vom Geschehen über Geschichte, Erzählung und Narration, verdoppeln sich vielmehr sofort und stehen sich gegenüber wie zwei Bilder einer Spielkarte. Darum erscheint selbst der modernen Narratologie, solange sie noch hermeneutisch konzipiert ist, das Geschehen immer schon als ein rätselhaftes semantisches Magma voller Bedeutungen, das sich durch seine narrative Figuration zunehmend in einen konkreten Sinn verwandelt.

Im Geschehen begegnet also auch aus dieser Sicht nicht einfach eine fiktive Tatsache, es begegnet immer schon eine realisierte Narration, deren metaphorische Form nur die Figuren in ihrer Erzählwirklichkeit für eine faktische Realität halten. In Wahrheit begegnen in den Ereignissen Metaphern. Deren Sichtbarkeit ist zwischen den Ansprüchen eines realistischen und allegorischen Erzählens, zumal mit Blick auf den späteren Artus- und Aventiureroman, durchaus skalierbar. Für das Erzählen ist aber gerade damit der generelle Anspruch der Metapher auch unabdingbar. Im Namen der narrativen Fiktionalität lässt sich diese elementare Metaphorizität nicht faktisch ausklammern. Denn es gibt kein Erzählen ohne Metapher, ebenso wie es keine Geschichte ohne Ereignis und keine Erzählung ohne Ereignishaftigkeit gibt.

In diesem Sinne ist auch die doppelte Selbsterfindung und Selbstfindung Iweins, einmal als Artusritter, der er sein will, und einmal als Löwenritter, zu dem er durch die Aventiure wird, eine metaphorische Antwort nicht nur auf das Fiktionsdilemma des Helden, sondern auch auf das Fiktionalitätsdilemma der Forschung. In der praktischen Interpretation der Erzählung durch die Erzählung, ebenso wie in der breiten Interpretationstradition des Iwein, die gleichsam durch den Erzähltext selbst lebendig gehalten wurde, ist darum das doppelt-gegenläufige Verhältnis der narrativen Ebenen immer schon wirksam und praktisch erkannt. In dieser Doppelrelation

\footnotetext{
21 Vgl. dazu die frühen Begriffsvorschläge bei Schmid, Wolf: »Die narrativen Ebenen >Geschehen<, >Geschichte<, >Erzählung< und >Präsentation der Erzählung««. In: Wiener slawistischer Almanach 9 (1982), S. 83-110; zusammenfassen Schmid, Wolf: Elemente der Narratologie. 3., erweiterte und überarbeitete Auflage. Berlin/Boston 2014; Bleumer, Hartmut: Art. »Historische Narratologie «. In: Christiane Ackermann/Michael Egerding (Hg.): Literatur- und Kulturtheorie in der Germanistischen Mediävistik. Ein Handbuch. Berlin/Boston 2015, S. 213-274.
} 
erscheint das Ergebnis des Erzählens zugleich immer auch als seine Voraussetzung. Und diese doppelte Relation ist durchaus nichts Postmodernes, sondern man wird es für einen typischen Mediävalismus, wenn nicht sogar für eine erzählphänomenologische Grundformel halten dürfen. ${ }^{22}$ Denn über die dynamisch-gegenläufige, doppelte Ebenenkorrelation bleibt das Spiel des Erzählens transhistorisch in Bewegung.

Iwein findet durch das Erzählen, indem er sich der Geschichte überlässt, eine ästhetische Lösung seines Identitätsproblems, die zugleich eine praktische ist. Sollte also nicht auch die Fiktionalitätsdiskussion durch eine historische Narratologie, die sich der doppelten, korrelativen Struktur der narrativen Selbstinterpretation mit ihrer semantisch so produktiven Paradoxie auch theoretisch bewusst ist, aus ihrem Dilemma herausfinden können?

Funding Open Access funding enabled and organized by Projekt DEAL.

Open Access Dieser Artikel wird unter der Creative Commons Namensnennung 4.0 International Lizenz veröffentlicht, welche die Nutzung, Vervielfältigung, Bearbeitung, Verbreitung und Wiedergabe in jeglichem Medium und Format erlaubt, sofern Sie den/die ursprünglichen Autor(en) und die Quelle ordnungsgemäß nennen, einen Link zur Creative Commons Lizenz beifügen und angeben, ob Änderungen vorgenommen wurden.

Die in diesem Artikel enthaltenen Bilder und sonstiges Drittmaterial unterliegen ebenfalls der genannten Creative Commons Lizenz, sofern sich aus der Abbildungslegende nichts anderes ergibt. Sofern das betreffende Material nicht unter der genannten Creative Commons Lizenz steht und die betreffende Handlung nicht nach gesetzlichen Vorschriften erlaubt ist, ist für die oben aufgeführten Weiterverwendungen des Materials die Einwilligung des jeweiligen Rechteinhabers einzuholen.

Weitere Details zur Lizenz entnehmen Sie bitte der Lizenzinformation auf http://creativecommons.org/ licenses/by/4.0/deed.de.

22 Zum Begriff des Mediävalismus als uneingesehener mediävistischer Denkfigur der Postmoderne vgl. Holsinger, Bruce: The Premodern Condition. Medievalism and the Making of Theory. Chicago 2005. 